\title{
EVALUATING RADIOMETRIC SENSITIVITY OF LANDSAT 8 OVER COASTAL/INLAND WATERS
}

\author{
Nima Pahlevan ${ }^{1,2^{*}}$, Jianwei Wei ${ }^{3}$, Crystal B. Schaaf ${ }^{3}$, John R. Schott ${ }^{4}$ \\ ${ }^{1}$ NASA Goddard Space Flight Center, Greenbelt, MD 20771, USA \\ ${ }^{2}$ Sigma Space Corporation, Lanham, MD 20706, USA \\ nima.pahlevan@nasa.gov \\ ${ }^{3}$ School for the Environment, University of Massachusetts at Boston, MA 02125, USA \\ Jianwei.Wei@umb.edu ,crystal.schaaf@umb.edu \\ ${ }^{4}$ Center for Imaging Science, Rochester Institute of Technology, Rochester, NY, 14623, USA \\ schott@cis.rit.edu
}

\begin{abstract}
The operational Land Imager (OLI) aboard Landsat 8 was launched in February 2013 to continue the Landsat's mission of monitoring earth resources at relatively high spatial resolution. Compared to Landsat heritage sensors, OLI has an additional 443- $\mathrm{nm}$ band (termed coastal/aerosol (CA) band), which extends its potential for mapping/monitoring water quality in coastal/inland waters. In addition, OLI's pushbroom design allows for longer integration time and, as a result, higher signal-to-noise ratio (SNR). Using a series of radiative transfer simulations, we provide insights into the radiometric sensitivity of OLI when studying coastal/inland waters. This will address how the changes in water constituents manifest at top-of-atmosphere (TOA) and whether the changes are resolvable at TOA (focal plane) relative to OLI's overall noise ${ }^{1}$.
\end{abstract}

Index Terms - OLI, Landsat 8, radiometric sensitivity, water constituents, water quality

\section{INTRODUCTION}

The Landsat program has provided the longest record of Earth Observation (EO) datasets amongst all of the existing EO programs. This valuable asset (which is freely released to the public) allows for regular monitoring of changes in the earth natural resources over the past four decades. The operational Land Imager (OLI) aboard Landsat 8 was

\footnotetext{
${ }^{1}$ This project was awarded (No. G12PC00072) by the United States Geological Survey (USGS) under the contract No. S20130000023292.
}

launched in February 2013 to continue the Landsat's mission for monitoring earth resources at relatively high spatial resolution. The enhancements include the addition of a 443- $n m$ channel and a 12-bit radiometric resolution.

The Landsat datasets, prior to the launch of Landsat 8, have been utilized in many studies for mapping water constituents. A few studies have demonstrated the potential of OLI in mapping optically active components (OACs) of water, including the near-surface concentrations of chlorophyll-a (CHL) and total suspended solids (TSS), as well as the absorption of the colored dissolved organic matter (CDOM), i.e., $\mathrm{a}_{\mathrm{CDOM}}$ [1, 2]. Fig. 1 illustrates the scene-derived signal-to-noise-ratio (SNR) curve obtained from $\sim 30$ recorded OLI scenes collected across different latitudes (solar zenith angle $\left(\theta_{\mathrm{s}}\right)$ ranging within $20^{\circ}-60^{\circ}$ ). The relatively high SNRs (for the CA and blue bands) comparable to those of coastal-ocean missions indicate promise for coastal/inland water studies.

Recognizing the potential of OLI for studying bio-optical properties in coastal/inland waters, we examine spectralradiometric (spectroradiometric) sensitivity of OLI for studying coastal/inland waters, i.e., how well changes in CHL, TSS, and $\mathrm{a}_{\mathrm{CDOM}}$ are detectable in the OLI's spectral bands, and how well the changes are resolvable at TOA (focal plane) relative to the system noise.

\section{APPROACH}

\subsection{METRIC}

Here, we introduce a realistic metric to measure the OLI's spectroradiometric (spectral-radiometric) sensitivity to 


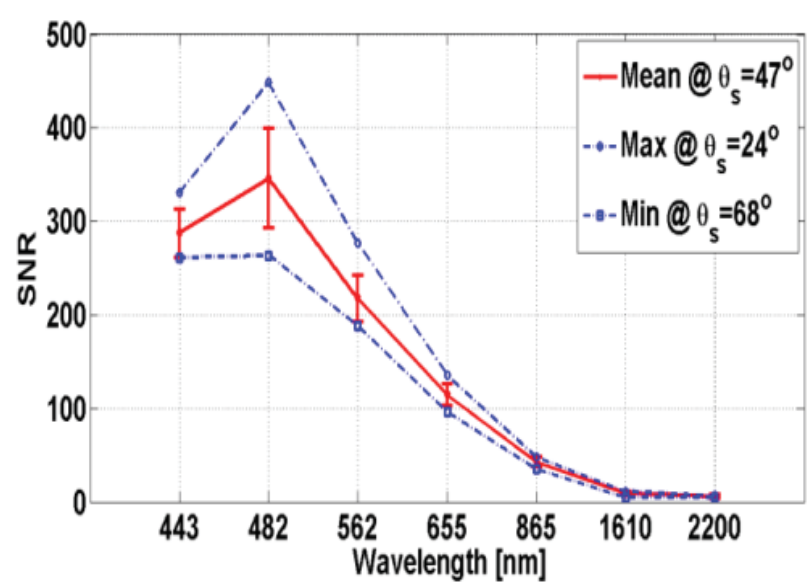

Fig. 1. The average scene-derived SNR $(n=30)$ calculated over uniform bodies of water. The dashed/dashed-dot curves indicate maximum and minimum SNRs derived from the scenes.

changes in water constituents in coastal/inland waters. The question to be answered is how well changes in OACs ( $\left.\triangle C H L, \triangle T S S, \triangle a_{C D O M}\right)$ can be discerned at TOA using the OLI instrument. From a radiometric standpoint, it is recognized that when the changes in the signal at TOA is less than the system noise level, the retrieved near-surface properties, e.g., water-leaving radiance; $L_{w}$, will bear significant uncertainties.

Here, we evaluate the (desired) signal versus the overall system noise for each band. The desired signal, is represented by the transmitted water-leaving radiance, i.e., $t L_{w}$, where $t$ is the diffuse attenuation for the sea-to-sensor path. The desired change in the signal (given the changes in CHL, TSS, and $\mathrm{a}_{\text {CDOM }}$ ) manifests in $t \Delta L_{w}$. From this term, the differential signal-to-noise ratio $(\Delta S N R)$ for the OLI's effective responses is defined as following [3]:

$\Delta \operatorname{SNR}\left(\lambda_{0} ; \Delta \mathrm{OAC}\right)=\frac{\left|\mathrm{t} \Delta \mathrm{L}_{\mathrm{w}}\right|}{\mathrm{N}}=\frac{\mathrm{t}\left|\mathrm{L}_{\mathrm{w}}^{\left(\mathrm{p}_{1}\right)}-\mathrm{L}_{\mathrm{w}}^{\left(\mathrm{p}_{2}\right)}\right|}{\mathrm{N}}$

where $\mathrm{N}$ is the effective system noise and represents the system overall noise due to photons, striping, quantization, electronics, etc. The $\Delta$ SNR metric, in fact, measures the signal contrast versus the overall system noise and is directly related to the noise equivalent change in radiance $(\mathrm{NE} \Delta \mathrm{L}) . \lambda_{0}$ denotes OLI's center wavelengths within the visible-near-infrared (VNIR) region at $443 \mathrm{~nm}, 482 \mathrm{~nm}, 562$ $\mathrm{nm}, 655 \mathrm{~nm}$, which represent CA, blue, green, and red bands, respectively. The differences in the water constituents ( $\triangle \mathrm{OAC}$ ) are modeled via Hydrolight [4] simulations for two adjacent pixels $\left(\mathrm{p}_{1}\right.$ and $\left.\mathrm{p}_{2}\right)$ in the scene located at an arbitrary location. The $\triangle \mathrm{OAC}$ parameter denotes a change in one of the constituents, i.e., $\triangle \mathrm{CHL}$, $\Delta \mathrm{TSS}$, or $\Delta \mathrm{a}_{\mathrm{CDOM}}(440)$. When a desired change in one component is desired, the two other components are held fixed. For example, a change in $\mathrm{CHL}(\Delta \mathrm{CHL})$ is estimated when TSS and $\mathrm{a}_{\text {CDOM }}(440)$ as well as the spectral shapes of the inherent optical properties (IOPs) remain constant [4]. The band-specific spectroradiometric sensitivity $(\delta O A C)$ of OLI is then estimated at $\Delta \mathrm{SNR}=1$, i.e., when $\mathrm{t} \Delta \mathrm{L}_{\mathrm{w}}=\mathrm{N}$ in Eq. 1. In essence, this defines a threshold (detection limit) below which detecting changes is limited by the instrument's spectral-radiometric capability. The detection limits are referred by $\delta \mathrm{CHL}$, $\delta \mathrm{TSS}$, and $\delta \mathrm{a}_{\text {СDOM }}(440)$, respectively. The terms in the numerator (Eq. 1) are simulated through radiative transfer modeling while the noise term in the denominator is derived from measurements of selected OLI scenes.

\subsection{SIMULATIONS}

In this study, water-leaving radiance $\left(L_{w}\right)$ for various water types is modeled with the Hydrolight code, whereas the effective noise is estimated through scene analysis.

The Hydrolight simulations were conducted for various CHL, TSS, and $\mathrm{a}_{\mathrm{CDOM}}(440)$ levels within the 0.2 to 10.0 $\mathrm{mg} / \mathrm{m}^{3}, 0.0$ to $12.0 \mathrm{~g} / \mathrm{m}^{3}$, and 0.05 to $2.0 \mathrm{~m}^{-1}$ ranges, respectively. The input concentrations span a reasonable range representing moderately productive, mesotrophic waters to highly turbid/trophic waters. The VNIR region (400 to $900 \mathrm{~nm}$ ) in-water simulations at $5 \mathrm{~nm}$ resolution were conducted for three solar geometries, i.e., $\theta_{\mathrm{s}}=$ $25^{\circ}, 45^{\circ}, 60^{\circ}$, and the Julian day of 270 (representing an average Earth-Sun distance). The Fournier-Forand phase function with $2 \%$ backscattering fraction $\left(b_{b} / b=0.02\right)$ was chosen for all the simulations . It should be noted that a moderate wind speed, i.e., $5 \mathrm{~m} / \mathrm{s}$, and aerosol optical thickness of 0.1 at $550 \mathrm{~nm}$ were used for the simulations. While it is desired to perform simulations with very fine OAC increments, a compromise between the computation time and the simulation step sizes was made: CHL was chosen to have increments of 0.2 units of concentrations, TSS was incremented by 0.1 units, and the reference CDOM absorption, i.e., $a_{C D O M}(440)=0.18 \mathrm{~m}^{-1}$, was scaled by multiplicative factors ranging from 0.3 to 11 with increments of 0.06 . This sampling scheme provides sufficiently fine-scale samples for properly analyzing OLI's spectroradiometric sensitivity. The Hydrolight-modeled water-leaving radiance $\left(L_{w}^{m}\right)$ were then propagated to the TOA using MODTRAN [5] to obtain simulated TOA radiance $\left(L_{t}^{m}\right)$ and $t L_{w}^{m}$. The TOA quantities were then passed through the OLI's RSR functions. Note that the relative spatial response (RSR) functions represent the cascade of the performance of all system components (e.g. quantization, optics, and electronics).

\subsection{SCENE-DEPENDENT NOISE}

To estimate the effective scene noise $(\mathrm{N})$ on the focal plane, the scene-derived standard deviations and mean TOA $\left(\mathrm{L}_{\mathrm{t}}\right)$ 


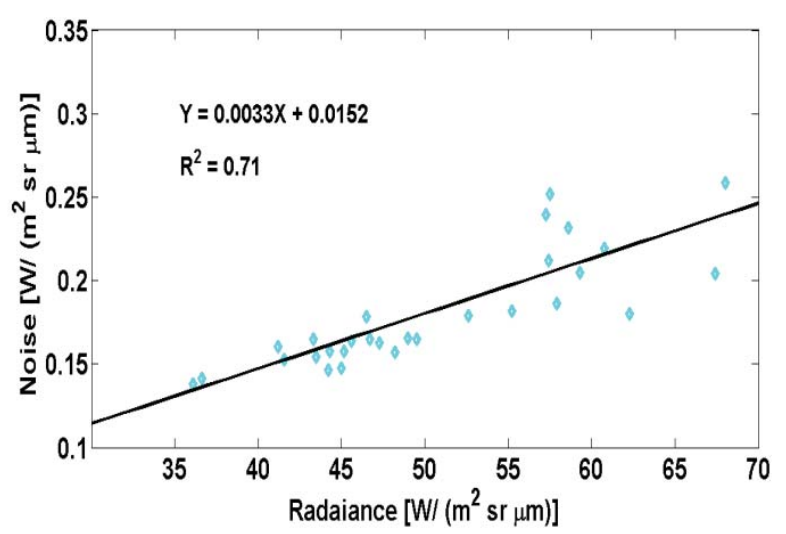

Fig. 2. The scene-derived noise plotted against TOA radiance for the CA band. The linear relationship provides a realistic estimate of system noise for a given TOA radiance (derived from OLI scenes).

radiances were calculated over spatially uniform bodies of waters, i.e., $10 \times 10$ pixels. The relationships constructed between $\mathrm{N}$ and $\mathrm{L}_{t}$ for OLI scenes $(\mathrm{n}=30)$ are used to deduce the noise level $(\mathrm{N})$ for the model-derived TOA radiances $\left(\mathrm{L}_{\mathrm{t}}^{\mathrm{m}}\right)$. Fig. 2 illustrates the observed signal-noise relationship for the CA bands. The diversity of the selected scenes for noise and signal estimation provides an extended dynamic range of observed total sensor-reaching radiances. This allows for evaluating the effective noise for various modeled TOA radiances. Linear regression analysis is applied to the modeled TOA radiances to estimate equivalent effective noise, and, as a result, $\Delta$ SNR (Eq. 1).

\section{RESULTS}

Many combinations of OACs were examined to map out OLI's spectroradiometric sensitivity to changes in OACs for a nominal solar zenith angle, i.e., $\theta_{s}=40^{\circ}$. Later, the impact of changes in $\theta_{s}$ will be discussed. Fig. 3 illustrates the band-specific $\mathrm{CHL}$ detection limits $(\delta \mathrm{CHL})$ for three different ranges of $\mathrm{CHL}$, i.e., $0.1<\mathrm{CHL} \leq 1,1<\mathrm{CHL} \leq$ 5 , and $5<\mathrm{CHL}<12$, referred to as case I, II, and III, respectively (not to confuse with widely used case I and case II water types). Realistic TSS and $\mathrm{a}_{\text {СDом }}(440)$ values in accordance with each CHL range were incorporated. It is revealed that for all cases, the blue band (482 $\mathrm{nm}$ ) shows the highest sensitivity to changes in CHL, i.e., on average, $\delta \operatorname{CHL}(482)>0.5 \mathrm{mg} / \mathrm{m}^{3}$. This implies difficulties in detecting changes smaller than 0.5 units CHL on the focal plane using this single band. The spectroradiometric sensitivity of the blue band degrades for the most trophic waters studied here $(5<\mathrm{CHL}<12)$. This applies to all OLI's bands. While the CA and the green bands, on average, exhibit similar sensitivity to the changes in CHL, the CA band is slightly better for case I (least trophic here) and case II (moderately trophic) waters. Nevertheless, in highly trophic waters, the green band shows lower detection

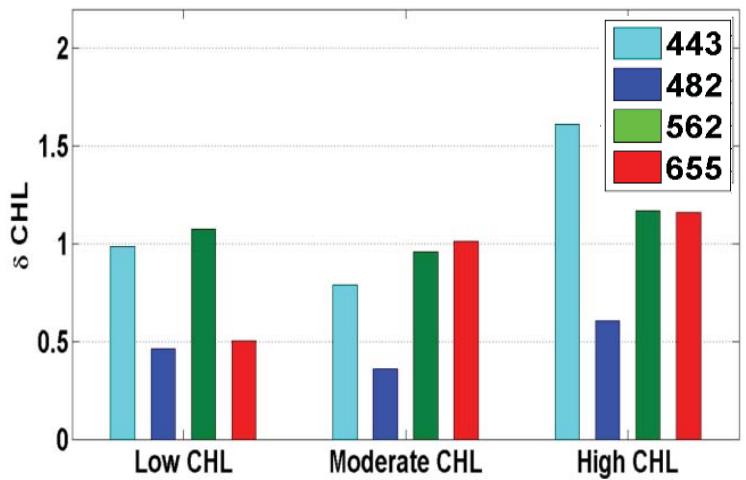

Fig. 3. The OLI's band-specific detection limits for CHL. The CA band $(443 \mathrm{~nm})$ is found as sensitive as the green band for low CHL conditions. Three CHL level are as following $0.1<\mathrm{CHL}<1$, $1<\mathrm{CHL}<5$, and $5<\mathrm{CHL}<12$. Note that the lower $\delta \mathrm{CHL}$ the more sensitive is OLI (relative changes in CHL).

limits. In relatively low-productivity waters, the red band is nearly as sensitive as the blue band $(\delta C H L(655) \sim 0.5)$, whereas it represents similar detection limits as in the green band for more turbid waters (case II and III).

The spectroradiometric sensitivity of the OLI bands with respect to the changes in TSS is shown in Fig. 4. The water types are classified into three broad regions, $0.1<$ TSS $<1$, $1<$ TSS $<5$, and $5<$ TSS $<12$. Compared to CHL, OLI's sensitivity relative to $\Delta$ TSS is more than $10 \times$ better than that for $\triangle \mathrm{CHL}$. As expected, the sensitivity diminishes, particularly in the CA band, for highly turbid waters (with high CDOM). The green band, on average, shows most sensitivity to changes in TSS. This is attributed to the relatively small total absorption within this region of the spectrum. Overall, the OLI sensor is expected to be capable of detecting differences in TSS larger than, on average, $0.1 \mathrm{~g} / \mathrm{m}^{3}$, i.e., $\delta \mathrm{TSS} \approx 0.1$, in different water types. The water types are classified into three broad regions: $0.1<$ TSS $\leq 1,1<$ TSS $\leq 5$, and $5<$ TSS $<12$. Compared to the sensitivity to CHL, the sensitivity with respect to TSS is found to be $10 \times$ better. As expected, the sensitivity diminishes, particularly in the CA band, for highly turbid waters (with high CDOM). The green band, on average, shows most sensitivity to changes in TSS. This is attributed to the relatively small total absorption within this region of the spectrum.

The detection limits associated with $\mathrm{a}_{\mathrm{CDOM}}(440)$ for the OLI's CA, blue, and green bands are illustrated in Fig. 5. The red band was found insensitive to the changes in $\mathrm{a}_{\mathrm{CDOM}}(440)$. In a similar fashion as for CHL and TSS, the water types were classified into three broad regions, $0.01<\mathrm{a}_{\text {СDOM }}(440) \leq 0.1,0.1<\mathrm{a}_{\text {CDOM }}(440) \leq 0.5$, and $0.5<\mathrm{a}_{\text {CDOM }}(440)<2$. In relatively low CDOM concentrations, i.e., $\mathrm{a}_{\text {CDOм }}(440)<0.5 \mathrm{~m}^{-1}$, with CHL and TSS remaining below 5 units, the blue and the green bands exhibit the highest sensitivity $\left(\delta \mathrm{a}_{\mathrm{CDOM}}(440)<0.1\right)$, 


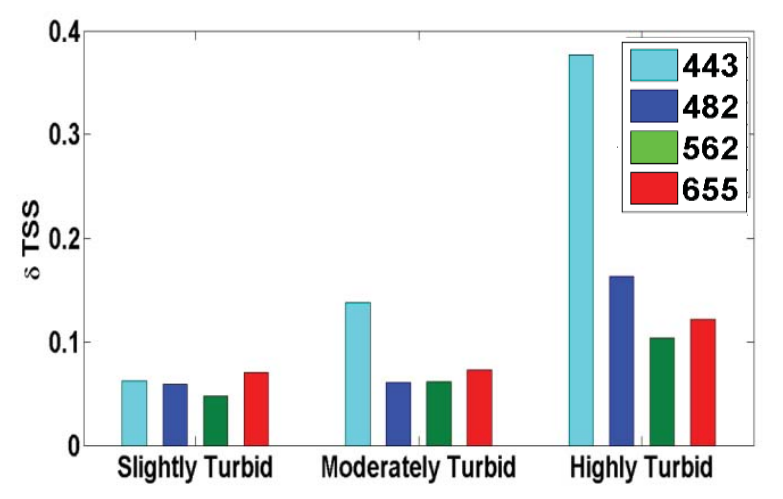

Fig. 4. The OLI's band-specific detection limits for TSS. The sensor is, on average, sensitive to $\Delta$ TSS $>0.1 \mathrm{~g} / \mathrm{m}^{3}$ in nearly all bands and for all water types. The CA band is found as sensitive as the other bands in slightly turbid waters.. Three TSS level are as following $0.1<$ TSS $<1,1<$ TSS $<5$, and $5<$ TSS $<12$.

whereas the sensitivity of the CA band is just above $0.15 \mathrm{~m}^{-1}$. The detection limit for CDOM-rich waters, i.e., $0.5<\mathrm{a}_{\text {СDом }}(440)<2$, deteriorates for the OLI's CA and blue bands (due to significant CDOM-induced absorption in the ultraviolet-blue portion of the spectrum). In such waters, the green band provides the best sensitivity to changes in $\mathrm{a}_{\text {CDOM }}(440)$, i.e., $\delta \mathrm{a}_{\text {CDOM }}(440) \sim 0.1$. One must bear in mind that the green band is also relatively highly sensitive to TSS and CHL and care must be taken when evaluating the responses in this band.

The detection limits can be improved $n \times$ by averaging the signal over $\mathrm{n} \times \mathrm{n}$ areas. The average detection limits of 0.5 units for CHL, i.e., $\delta \mathrm{CHL}=0.5 \mathrm{mg} / \mathrm{m}^{3}$, for instance, will be enhanced to $\delta \mathrm{CHL}=0.167 \mathrm{mg} / \mathrm{m}^{3}$, if a $3 \times 3$ smoothing kernel is applied on the recorded OLI datasets. One must balance the loss of spatial details against the gain in the retrieval sensitivity in areas where spatial variability in OACs is deemed minimal, i.e., smoothing will improve retrieval performance. For high-latitude OLI observations (not presented here), where $\theta_{s} \approx 60^{\circ}$, our analysis indicates that there is, on average, a $30 \%$ reduction in the detection limits; on the other hand, when $\theta_{s}=25^{\circ}$, the radiometric sensitivity is, on average, only improved $\sim 6 \%$, when compared to the mid-latitude detection limits (Figs. 3,4, and 5). Note that the $\triangle S N R$ metric is intended as an engineering tool to assess the expected performance limits. Actual retrievals will be improved by the use of multiple bands and may be degraded by calibration errors and less ideal atmospheric compensation technique.

\section{CONCLUSION}

The analysis of the spectroradiometric sensitivity of OLI using $\triangle S N R$ as metric gives clues on the errors anticipated for the retrieval of the biogeochemical properties (CHL,

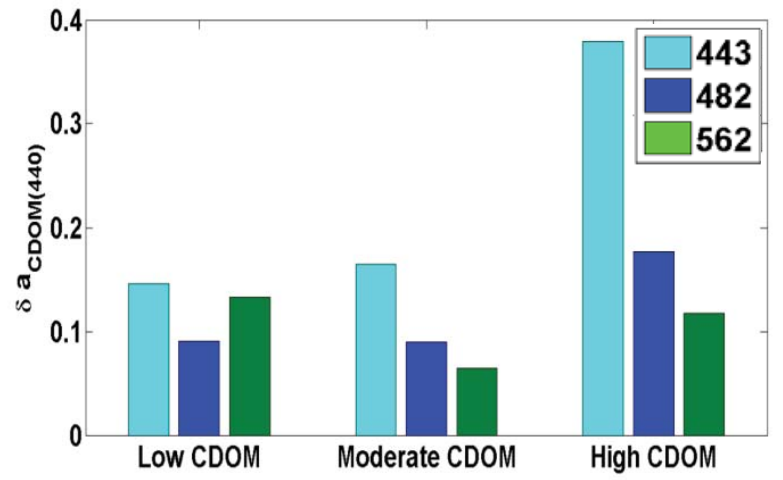

Fig. 5. The OLI's band-specific detection limits for $a_{C D O M}(440)$. The sensor is, on average, sensitive to $a_{C D O M}(440)>0.1 \mathrm{~m}^{-1}$ for water types with $a_{C D O M}(440)<0.5 \mathrm{~m}^{-1}$. The red band is excluded from this chart due to its insensitivity to changes in CDOM. Three CDOM levels are as $0.01<a_{\text {CDOM }}(440) \leq 0.1$, $0.1<a_{C D O M}(440) \leq 0.5$, and $0.5<a_{C D O M}(440)<2$.

TSS, $\left.\mathrm{a}_{\mathrm{CDOM}}\right)$. For an average solar zenith angle $\left(\theta_{\mathrm{s}}=45^{\circ}\right)$, it was found that OLI is, on average, sensitive to changes in CHL, TSS, and $\mathrm{a}_{\text {CDOM }}(440)$ larger than $0.5 \mathrm{mg} / \mathrm{m}^{3}$ and $0.1 \mathrm{~g} / \mathrm{m}^{3}$, and $0.1 \mathrm{~m}^{-1}$, respectively. For high-latitude areas with $\theta_{\mathrm{s}} \sim 60^{\circ}$, the sensitivity degrades up to $30 \%$. To improve the retrieval of OACs over large bodies of waters, the OLI scenes may be smoothed to lower the noise contribution. This study demonstrated OLI's band-specific sensitivity to changes in OACs, despite its relatively broad spectral bands (when compared to the corresponding ocean channels of MODIS and those of VIIRS). In addition to limitations in the OLI's radiometric sensitivity, further complications are introduced when correcting for the atmospheric effects.

\section{REFERENCES}

[1] A. D. Gerace, et al., "Increased potential to monitor water quality in the near-shore environment with Landsat's next-generation satellite," Journal of Applied Remote Sensing, vol. 7, pp. 073558073558, 2013.

[2] N. Pahlevan and J. R. Schott, "Leveraging EO-1 to Evaluate Capability of New Generation of Landsat Sensors for Coastal/Inland Water Studies," Selected Topics in Applied Earth Observations and Remote Sensing, IEEE Journal of, vol. 6, pp. 360-374, 2013.

[3] N. Pahlevan, et al., "Diurnal remote sensing of coastal/oceanic waters: a radiometric analysis for Geostationary Coastal and Air Pollution Events," Applied Optics, vol. 53, pp. 648-665, 2014/02/01 2014.

[4] C. D. Mobley, Sundman, L.K., "Hydrolight 5, Ecolight5 User Guide," Sequoia Scientific, Inc., Bellevue2008.

[5] A. Berk, et al., "MODTRAN5: 2006 update," pp. 62331F-62331F, 2006. 